\title{
Formation of porous networks on polymeric surfaces by femtosecond laser micromachining
}

\author{
Youssef Assaf, Anne-Marie Kietzig* \\ Department of Chemical Engineering, McGill University, 3610 University Street, \\ Montréal, Québec, Canada, H3A 0C5
}

\begin{abstract}
In this study, porous network structures were successfully created on various polymer surfaces by femtosecond laser micromachining. Six different polymers (poly(tetrafluoroethylene) (PTFE), poly(methyl methacrylate) (PMMA), high density poly(ethylene) (HDPE), poly(lactic acid) (PLA), poly(carbonate) (PC), and poly(ethylene terephthalate) (PET)) were machined at different fluences and pulse numbers, and the resulting structures were identified and compared by lacunarity analysis. At low fluence and pulse numbers, porous networks were confirmed to form on all materials except PLA. Furthermore, all networks except for PMMA were shown to bundle up at high fluence and pulse numbers. In the case of PC, a complete breakdown of the structure at such conditions was observed. Operation slightly above threshold fluence and at low pulse numbers is therefore recommended for porous network formation. Finally, the thickness over which these structures formed was measured and compared to two intrinsic material dependent parameters: the single pulse threshold fluence and the incubation coefficient. Results indicate that a lower threshold fluence at operating conditions favors material removal over structure formation and is hence detrimental to porous network formation. Favorable machining conditions and material-dependent parameters for the formation of porous networks on polymer surfaces have thus been identified.
\end{abstract}

Keywords: Femtosecond laser, micromachining, polymer, microstructure, porosity, lacunarity analysis

\section{INTRODUCTION}

Polymers have been extensively used as biomaterials due to their desirable bulk properties such as elasticity and degradability ${ }^{1}$. However, applications that require enhanced cell attachment and growth such as stents ${ }^{2}$ and grafts ${ }^{3}$ are heavily dependent on surface properties. High porosity, enhanced surface area, and multi-scale roughness are desirable for such applications, and significant effort has been made in order to impart those properties to polymeric surfaces ${ }^{4}$. Lithography, which is the current industry standard for polymer surface patterning and modification, only partially achieves that goal. While it successfully enhances surface area, achieving hierarchical roughness requires a multi-step process involving iterative layer-by-layer coating and exposure that does not typically induce porosity ${ }^{5}$.

With the emergence of femtosecond (fs) technology, laser micromachining is now able to introduce dual scale roughness (both micro- and nano-) on target surfaces ${ }^{6}$. Furthermore, it is a single-step, non-contact method that delivers energy in pulses of such small duration that heat-induced damage to the material is minimized ${ }^{7}$.

Ultrashort laser-polymer interaction is still under investigation as many photochemical ${ }^{8}$ and non-linear optical processes $^{9}$ are involved. As a result, most of the current literature concerning fs polymer machining focuses on hole or line ablation in the aim of studying local polymer interaction with a select number of laser pulses as opposed to machining over a large area. However, laser-induced porosity on polymer surfaces has been observed on the bottom or side walls of inscribed craters and lines in the past. For example, fibre-like structure formation on the side walls of PTFE ablation craters has been reported in several studies ${ }^{10-12}$. In addition, several publications have reported the appearance of micro-pores on $\mathrm{PMMA}^{13-15}$ and $\mathrm{PC}^{16}$ after fs laser hole and line ablation.

*Corresponding author. Tel: +1(514) 398-3302. e-mail: anne.kietzig@mcgill.ca 
This aspect of polymer laser ablation has been mostly reported as an unsuspected side effect of machining and it is unclear how it would affect surface topology when machining over an area. Baset et. al's (2013) recent study of PMMA ablation is one of the few publications focusing on fs laser induced porosity on polymer surfaces ${ }^{13}$. Their results have shown that the porous area fraction is dependent on pulse energy as well as number of pulses for hole ablation and scanning speed for line ablation. This type of porosity could therefore be controllable by adjusting machining parameters. Recently, Liang et al. (2014) successfully produced a novel porous network structure on PTFE by fs laser micromachining over a large area ${ }^{17}$. Their machining technique led to the formation of a fibrillary network that gets increasingly entangled as a function of fluence. However, very little is known about the formation mechanisms or even the properties of such structures. In addition, no information about the depth over which these structures form is given. Furthermore, it has not yet been established if this type of network can be reproduced on other polymers which might be more suitable for in-vivo use than PTFE.

In summary, fs laser induced micro-porosity has been observed on several polymers. In the case of PTFE, it leads to the formation of a porous network structure when homogeneously machining the surface over an area. This study aims at studying fs laser area machining of a variety of polymers in order to identify which machining conditions and materialdependent parameters are favorable for the formation of porous networks.

\section{MATERIALS AND METHODS}

\subsection{Materials:}

The following six polymers were studied: poly(tetrafluoroethylene) (PTFE), poly(methyl methacrylate) (PMMA), high density poly(ethylene) (HDPE), poly(lactic acid) (PLA), poly(carbonate) (PC), and poly(ethylene terephthalate) (PET). For each material, 3 by $3 \mathrm{~mm}$ square samples were cut out from a commercially available sheet (McMaster-Carr). The samples were $1.5 \mathrm{~mm}$ thick with the exception of PLA $(0.9 \mathrm{~mm})$ and PTFE $(3 \mathrm{~mm})$.

\subsection{Laser micromachining:}

The samples were micromachined using an amplified Ti:sapphire solid-state laser (Coherent Libra) which emits a horizontally polarized Gaussian beam of $800 \mathrm{~nm}$ wavelength, $4 \mathrm{~W}$ output power, and in pulses of $85 \mathrm{fs}$ duration. It was operated at a repetition rate of $1 \mathrm{kHz}$. The beam power is controlled through a variable attenuator which consists of a halfwave plate and a polarizing beam splitter. The beam is then focused using a $100 \mathrm{~mm}$ plano-convex lens down to a theoretical $1 / e^{2}$ spot size of $44 \mu \mathrm{m}$ onto a motorized 3D translational stage (Newport Corporation) which is subjected to the stationary laser beam. The stage's movement and velocity were digitally controlled via the GOL3D software (GBC\&S) along with a mechanical shutter (Uniblitz) in order to create different machining patterns. Lines were machined by moving the stage in the horizontal direction while patches were created by vertically overlapping lines in a raster scan pattern. The samples' surfaces were placed at the focal plane of the lens. Immediately after machining, all samples were placed in a sonication ethanol bath for five minutes to remove any loose machining debris.

\subsection{Machining parameters:}

Two types of machining experiments were performed. Line scans for the determination of material dependent parameters and raster scans for microstructure identification. The pulse peak fluence $F_{0}$ and pulse-per-spot number $P P S$ were used as variable parameters. The latter was calculated as follows:

$$
P P S=\left(\frac{\omega_{0} f_{p}}{v}\right)\left(\frac{1}{1-\Phi}\right) .
$$

Where $\omega_{0}$ represents the theoretical beam diameter, $f_{p}$ the laser's repetition rate, $v$ the scanning velocity and $\Phi$ the vertical overlap between two raster scanned lines (equal to zero for a line scan).

For each polymer, 40 lines were machined at the conditions corresponding to all the possible combinations of $F_{0}$ and PPS presented in Table 1. 
Table 1. Line ablation machining parameters

\begin{tabular}{cc|cc}
\hline \multirow{2}{*}{ Power $(\mathbf{m W})$} & Corresponding $\boldsymbol{F}_{\mathbf{0}}\left(\mathbf{J} / \mathbf{c m}^{\mathbf{2}}\right)$ & $\boldsymbol{v}(\mathbf{m m} / \mathbf{s})$ & Corresponding $\boldsymbol{P P S}$ \\
\hline & 0.2 & 0.22 & 200 \\
4.5 & 0.6 & 2.2 & 20 \\
4.4 & 1.3 & 4.4 & 10 \\
9.9 & 2.2 & 33 & $1^{\mathrm{a}}$ \\
17 & 3.3 & & \\
25 & 4.5 & & \\
34 & 5.8 & & \\
44 & 7.2 & & \\
54 & 8.5 & & \\
76 & 10 & & \\
\hline
\end{tabular}

a-

This velocity actually corresponds to a PPS number of 1.3 and was the highest velocity achievable with our setup. However, this condition was considered equal to a PPS of 1 since all of the presented results were measured at the center of an ablated hole which is unaffected by the pulse overlap at the hole's edges.

In addition, 30 patches were machined on each of the six polymers corresponding to a combination of $10 F_{0}$ values (same as Table 1) and $3 P P S$ values (listed in Table 2).

Table 2. PPS parameters for patch ablation

\begin{tabular}{ccc}
\hline $\boldsymbol{v}(\mathbf{m m} / \mathbf{s})$ & $\boldsymbol{\Phi}$ & $\boldsymbol{P P S}$ \\
\hline & & \\
8 & 0.95 & 220 \\
16 & 0.95 & 55 \\
\hline
\end{tabular}

All of these conditions were confirmed to result in a homogeneous fluence profile according to the accumulated fluence profile (AFP) model following a procedure outlined in Ahmmed et al.'s (2015) recent work. ${ }^{18}$.

\subsection{Topography imaging, measurements, and analysis:}

Line width was measured by 3D confocal microscopy (Olympus LEXT OLS4000). The surface topography was imaged via scanning electron microscopy (SEM) (FEI Inspect F50). This required coating the samples with a $10 \mathrm{~nm}$ thick layer of gold by low-vacuum sputtering in order to avoid surface charging. SEM micrographs were quantitatively analyzed by using the Fraclac plugin for the ImageJ image analysis software ${ }^{19}$. 


\subsection{Microstructure thickness determination:}

The micromachined square patches of interest were cut in half perpendicularly to scanning direction with a diamond saw blade. The resulting cross-section was polished with 1200 grit silicon carbide sandpaper. The depth of removed material and the microstructure thickness was then determined by imaging with an environmental scanning electron microscope (FEI Quanta 450).
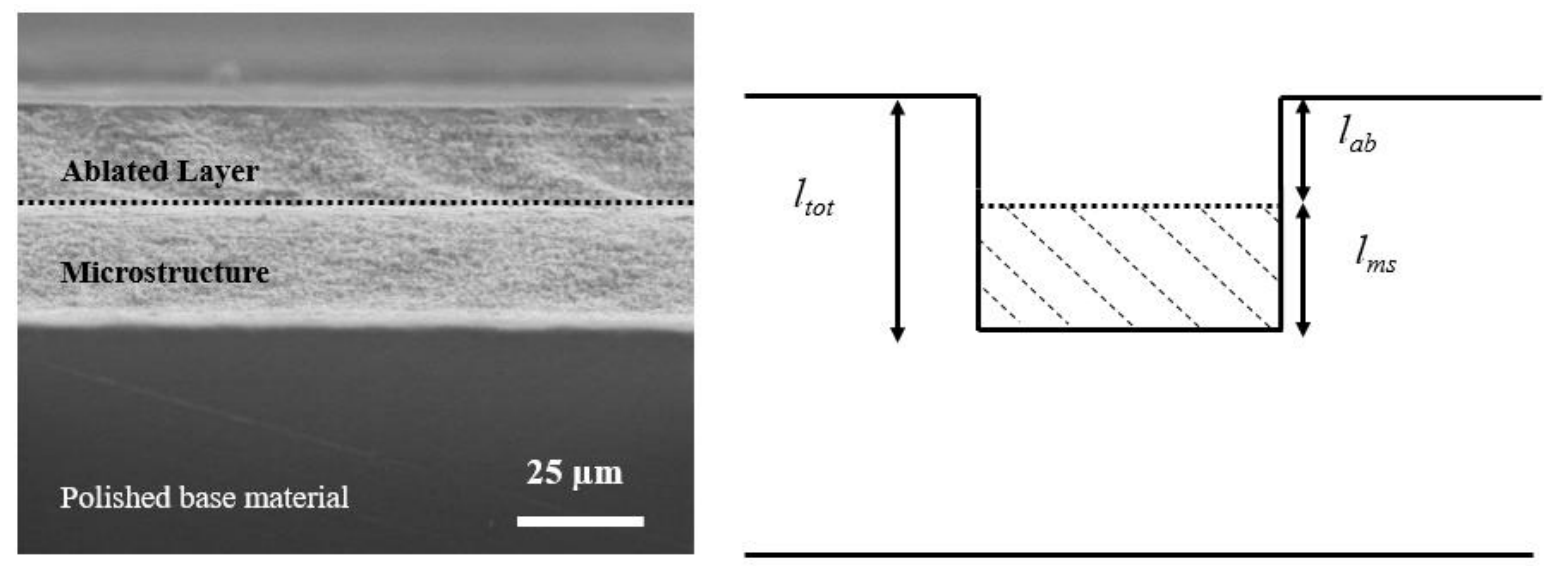

Figure 1. Microstructure thickness determination

As seen in Figure 1, two thicknesses were measured: $l_{a b}$ which represents the thickness of the layer of ablated material, and $l_{m s}$ which represents the thickness of the microstructure itself. Their sum $l_{\text {tot }}$ represents the total depth of affected material.

\subsection{Lacunarity analysis:}

Microstructure identification and comparison is usually qualitative and involves a visual assessment of SEM micrographs. In this study, the presented microstructures are quantitatively compared by lacunarity measurements on their SEM images. Lacunarity is typically calculated by the sliding-box counting algorithm ${ }^{20}$, in which a box of dimension $r^{*} r$ slides across a binary image. The number of foreground pixels in that box is measured at each position along the image and a distribution is generated. The lacunarity $\lambda$ of the image can then be calculated from this distribution:

$$
\lambda(r)=\left(\frac{s_{k}(r)}{\bar{k}(r)}\right)^{2}+1 .
$$

where $s_{k}$ and $\bar{k}$ represent the standard deviation and mean of the foreground pixel distribution at a given box size $r$. A higher lacunarity value means an image is spatially coarse or clumped while a lower value corresponds to a fine texture. From equation 6 , it is evident that $\lambda$ is highly dependent on the box size $r$. Therefore, when comparing two SEM images, looking at lacunarity values at a single $r$ does not necessarily reflect the overall texture. One way to generate a single lacunarity parameter is to calculate the area under the $\ln (\lambda)$ vs. $\ln (r)$ curve $^{21}$ :

$$
\Theta=\int_{0}^{\ln M} \ln (\lambda) \operatorname{dln}(r) .
$$

where $M$ corresponds to the smaller dimension of the image being analyzed and $\Theta$ is termed as the spatial heterogeneity index. Recent publications have shown that $\Theta$ allows for the objective characterization and comparison of hierarchical surface topographies ${ }^{22-23}$. This approach was therefore used in this study.

SEM micrographs at 10,000x magnification were taken for every machined patch before being converted into binary images for lacunarity analysis. The conversion was performed by first equalizing the images' histograms and then applying a threshold of 0.5 to ensure the same amount of foreground and background pixels. This method is evidently dependent on the focus and contrast of the images in question so substantial effort was made in order to minimize these effects by collecting micrographs of similar quality. After the conversion was made, the $\lambda(r)$ function and the associated $\Theta$ were calculated for every image. 


\section{RESULTS AND DISCUSSION}

Figure 2 exemplarily highlights the microstructures machined at different $F_{0}$ at a $P P S$ number of 55 .

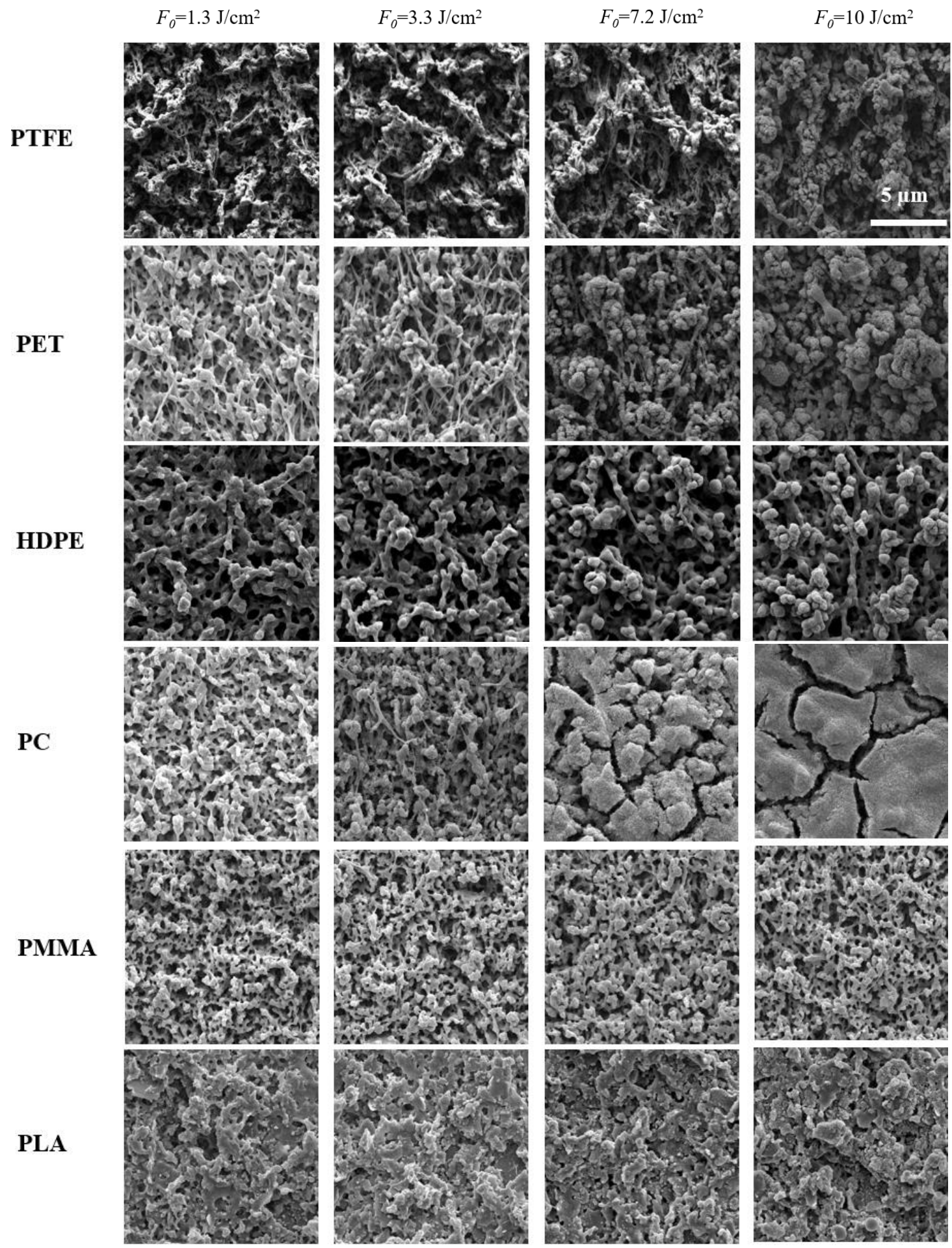

Figure 2. Microstructure topologies at $P P S=55$ 
At low fluence $\left(F_{0}=1.3 \mathrm{~J} / \mathrm{cm}^{2}\right)$, all of the materials except PLA form a porous network structure. In contrast, machining only seems to impart a randomly distributed roughness on PLA which does not noticeably change with fluence. For the rest of the materials, a large divergence in behaviors can be observed at high fluence $\left(F_{0}=10 \mathrm{~J} / \mathrm{cm}^{2}\right)$. PMMA seems to be the least affected by increasing fluence since the microstructure remains almost identical. HDPE, PTFE, and PET exhibit an increasingly larger degree of bundling of the network structure which leads to an apparent loss in porosity. PC does show some degree of bundling at intermediate fluences but quickly observes a complete breakdown of the network which transitions into a blocky structure. All of these trends were numerically validated through lacunarity analysis. Figure 3 illustrates the evolution of the lacunarity function exemplarily for PET as a function of fluence at a PPS number of 55 .

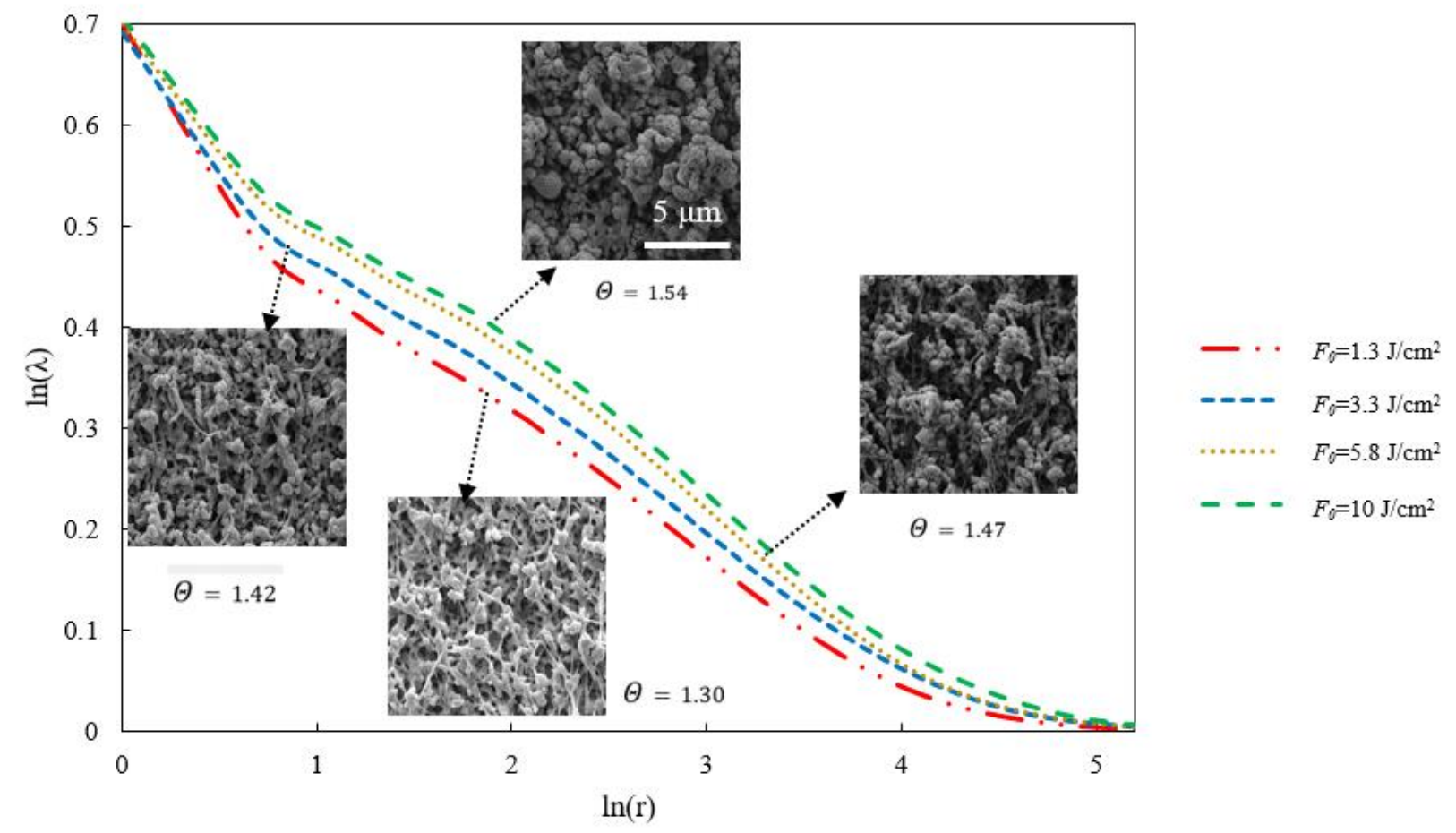

Figure 3. Lacunarity curves at different fluences for PET at $P P S=55$.

The plot clearly displays an increase in spatial heterogeneity for PET as a function of fluence. Indeed, the spatial heterogeneity index steadily increases from $\Theta=1.3$ at $F_{0}=1.3 \mathrm{~J} / \mathrm{cm}^{2}$ to $\Theta=1.54$ at $F_{0}=10 \mathrm{~J} / \mathrm{cm}^{2}$. This corresponds to a texture that is becoming increasingly coarse or clumped which actually confirms our observation of the structure bundling up.

Intra-material comparisons can be easily made since it is straightforward to obtain images that have similar contrast and brightness within the same material. However, inter-material comparisons are less effective. Even if similar focus and contrast can be achieved, the information depth of the pixels will be different due to the properties of the polymers, their interaction with the SEM beam, and the different topologies studied. Therefore, even if some microstructures exhibit similar trends with respect to fluence across materials, their spatial heterogeneity indices will not be comparable. For this reason, for every material, all of the indices were normalized with respect to the index corresponding to the lowest fluence where ablation occurred $\left(F_{0}=1.3 \mathrm{~J} / \mathrm{cm}^{2}\right.$ for all polymers). The evolution of the normalized index $\|\Theta\|$ as a function of fluence can therefore be compared for all materials while eliminating the effects mentioned above (Figure 4a). 

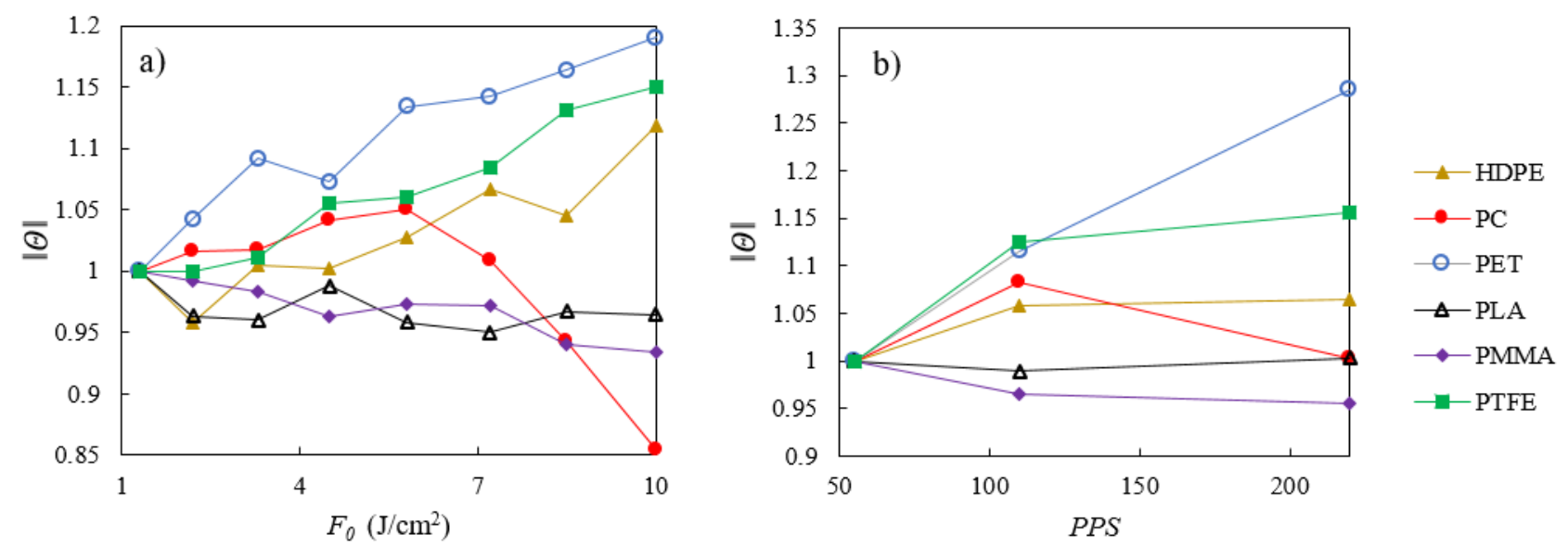

Figure 4. a) Normalized spatial heterogeneity index as a function of fluence at $P P S=55$. b) Normalized spatial heterogeneity index as a function of $P P S$ at $F_{0}=3.3 \mathrm{~J} / \mathrm{cm}^{2}$. The dashed lines only serve as visual guidelines.

This approach confirms our previous qualitative assessment of the microstructure. The bundling of the structure with fluence for HDPE, PTFE, and PET is translated into a steady increase in the heterogeneity index. Furthermore, the plot also demonstrates that the degree of bundling is higher for PET than for PTFE which in turn is higher than that of HDPE. PC follows a similar trend up to $F_{0}=5.8 \mathrm{~J} / \mathrm{cm}^{2}$. At higher fluences, the transition into the blocky structure translates into a very sharp decrease in $\Theta$. Finally, the stability of the microstructure with respect to fluence for PLA and PMMA is also confirmed as the normalized indices for these two materials hover between the values of 1 and 0.95 (albeit with a slightly decreasing trend). The agreement between our assessment of the SEM images and the quantitative data lead us to conclude that lacunarity analysis is a tool that can objectively characterize and compare fs laser induced microstructures on polymeric surfaces.

All of the results presented so far have been for a fixed PPS number of 55. The reason for that is that the effect of PPS on microstructure was observed to be extremely similar to the effect of fluence. Figure $4 \mathrm{~b}$ shows the evolution of lacunarity data with increasing $P P S$ numbers for $F_{0}=3.3 \mathrm{~J} / \mathrm{cm}^{2}$. For this plot, the indices were normalized by the value at $P P S=55$ for each material. By comparison with figure $4 \mathrm{a}$, it is evident that pulse number has the same qualitative effect on microstructure as fluence.

In conclusion, this parametric study shows that, with the exception of PMMA, operation at high fluence and pulse numbers is detrimental to porous network formation as it leads to bundling and increased structure coarseness or even complete structure breakdown in the case of PC. Therefore, operation slightly above a polymer's threshold fluence is recommended when aiming to avoid the abovementioned phenomena. PMMA's singular behavior indicates that one of its intrinsic material properties renders it resistant to structure bundling at high fluence. Its outstanding resistance to photooxidation $^{24}$ might indicate that porous network breakdown is related to photo-reactive processes.

After the identification of the different topologies, the depth over which the microstructures have formed was measured according to the procedure described in section 2.5. Figure 5 displays the results for PC at $P P S=55$. 


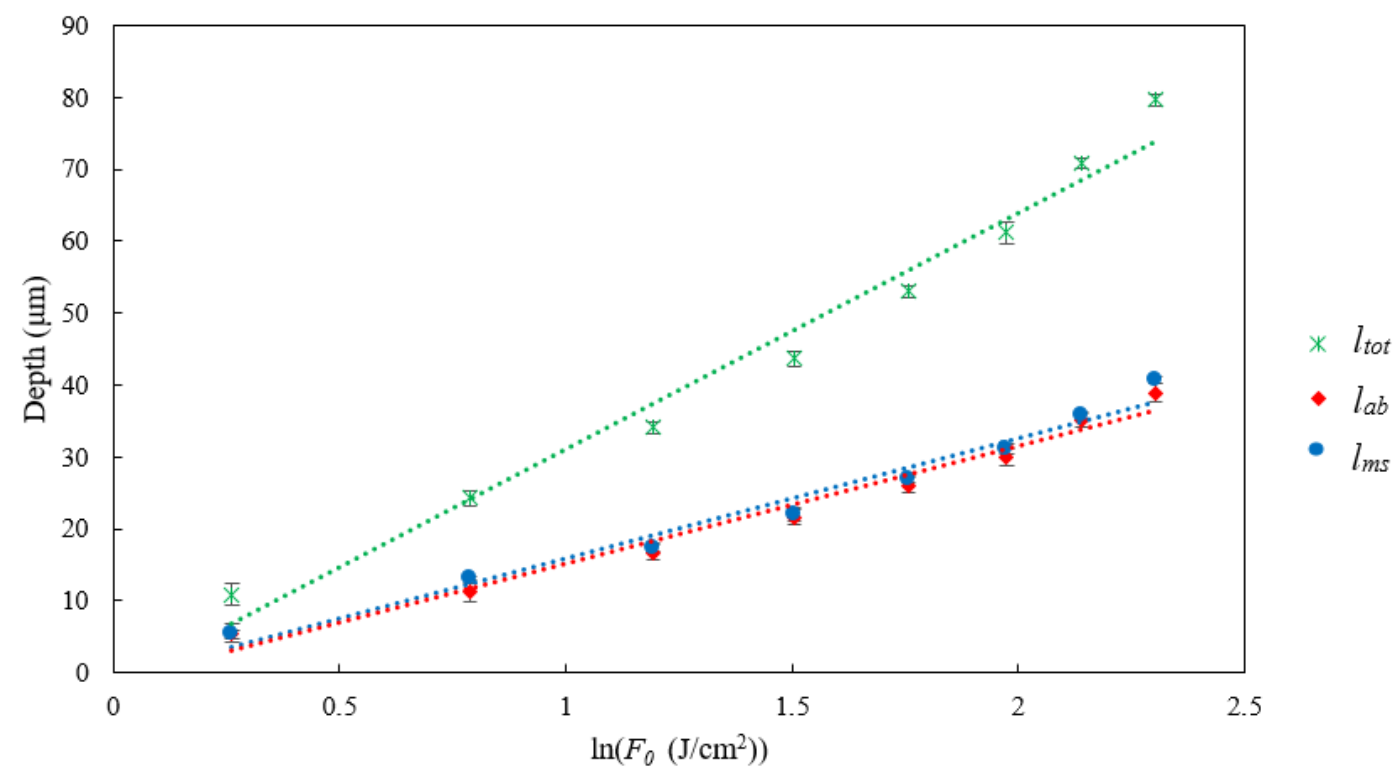

Figure 5. Modified depth as a function of fluence for PC patches at $P P S=55$. The error bars represent the standard deviation of three measurements.

As can be seen, both the thickness of the microstructure $l_{m s}$ and the ablated thickness $l_{a b}$ linearly increase as a function of $\ln \left(F_{0}\right)$. For all materials, $l_{m s}$ values ranged between 3 and $50 \mu \mathrm{m}$ within the range of our experiments. Furthermore, microstructure formation seems to occur over a length comparable to material ablation for all conditions as $l_{m s}$ and $l_{a b}$ are almost equal and correspond to approximately half of $l_{t o t}$. In other words, the average ratio of $l_{a b}$ over $l_{t o t}$ shows whether material removal or microstructure formation is the dominant mechanism for each material. As can be seen in Table 3, HDPE, PMMA, and PTFE displayed a similar behavior to PC with their $\overline{l_{a b} / l_{\text {tot }}}$ ratio being close to 0.5. The only materials where one of the mechanisms was dominant over the other were PLA and PET. For these two polymers, material is removed over a thickness that is significantly larger than the thickness of the microstructure formed. This discrepancy across materials can be explained by their threshold fluence at operating conditions $\left(F_{t h}\right.$ at $\left.P P S=55\right)$ which is also found in Table 3.

Table 3. Ablation Parameters and extent of material removal

\begin{tabular}{ccccc}
\hline Material & $\overline{\boldsymbol{l}_{\boldsymbol{a b}} / \boldsymbol{l}_{\text {tot }}}$ & $\boldsymbol{F}_{\boldsymbol{t h}}(\mathbf{1})\left(\mathbf{J} / \mathbf{c m}^{\mathbf{2}}\right)$ & $\xi$ & $\boldsymbol{F}_{\boldsymbol{t h}}(\mathbf{5 5})\left(\mathbf{J} / \mathbf{c m}^{\mathbf{2}}\right)$ \\
\hline & & & & \\
PLA & 0.62 & 1.24 & 0.62 & 0.28 \\
PET & 0.60 & 2.15 & 0.62 & 0.42 \\
PC & 0.53 & 1.83 & 0.73 & 0.60 \\
HDPE & 0.51 & 1.25 & 0.85 & 0.67 \\
PMMA & 0.49 & 2.50 & 0.74 & 0.89 \\
PTFE & 0.47 & 2.11 & 0.82 & 1.01 \\
& & & & \\
\hline
\end{tabular}




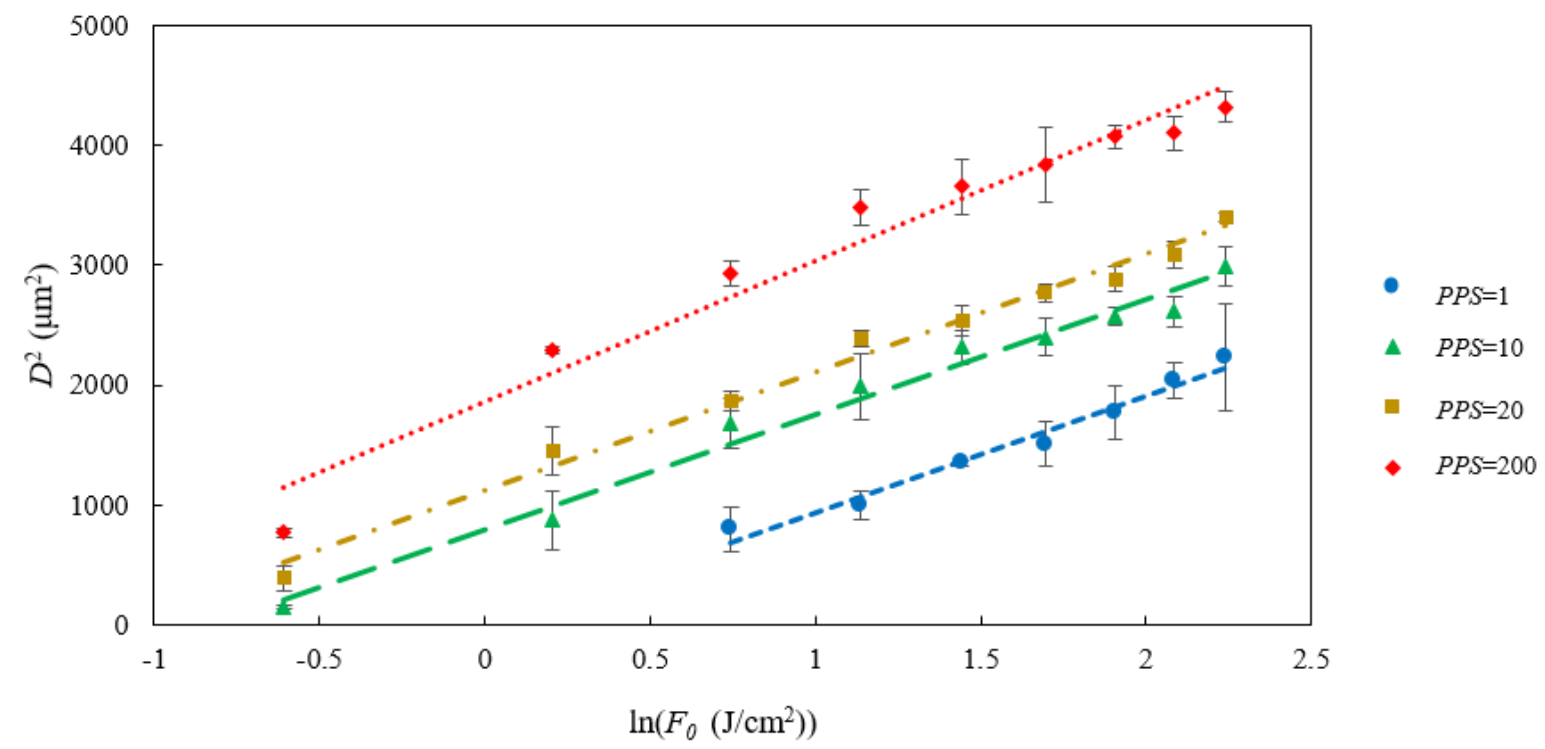

Figure 6: Line width results for PLA. The error bars represent the standard deviation of three measurements. The dashed lines represent the best fit of equation 4 .

The threshold fluence $F_{t h}$ at each PPS was determined by fitting line width data $(D)$ to Liu et al.'s model ${ }^{25}$ :

$$
D^{2}=\frac{1}{2} \omega_{0}^{2} \ln \left(\frac{F_{0}}{F_{t h}(P P S)}\right) .
$$

The incubation model first introduced by Jee et al. ${ }^{26}$ then allowed us to determine the single pulse $F_{t h}(1)$ value as well as the incubation coefficient $\xi$ (Table 3 ):

$$
F_{t h}(P P S)=F_{t h}(1) P P S^{\xi-1} .
$$

Figure 6 presents the results for PLA. The threshold fluence at 55 PPS, which corresponds to the actual machining conditions for area modification, was then calculated using equation 5 (Table 3). By comparing this parameter to the average ratio of $l_{a b}$ over $l_{t o t}$, we can clearly see that the extent of material removal is correlated to the threshold fluence at operating conditions. Indeed, materials with a high single pulse threshold fluence such as PMMA, a high incubation coefficient such as HDPE, or a combination of both such as PTFE have a higher threshold fluence at 55 PPS and are therefore less susceptible to material removal. On the other hand, PLA and PET both displayed an extremely low incubation coefficient which led to a very low threshold fluence at 55 PPS. In the specific case of PLA, the resulting threshold fluence is significantly lower than for all other polymers. As a result, even when operating at low fluence, almost all of the incoming energy can be converted into material ablation. This explains why no significant structure formation was observed on PLA using our machining technique. And since homogeneous area machining typically requires operation at high overlap and therefore high $P P S$, having a low incubation coefficient is detrimental to porous network formation since it will lead to a lower threshold fluence at operating conditions and limited structure formation.

\section{CONCLUSION}

The microstructures formed on six different polymers after femtosecond laser area machining were studied. Patches were machined on all polymers at several fluence and pulse number conditions. Lacunarity analysis identified several behaviors. Porous network structures were confirmed to form at low fluence on all polymers except PLA. PET, PTFE, and HDPE observed a bundling up of their structure with fluence. PC observed the same type of behavior in addition 
to the formation of a cracked structure at high fluence. PLA exhibited no microstructure formation. The evolution of microstructure as a function of pulse number followed the same trends. Operation at low pulse numbers and slightly above the threshold fluence is therefore recommended for porous network formation. The evolution of microstructure depth with respect to fluence showed that its extent is dependent on the material's threshold fluence at operating conditions. The latter was calculated by gathering the single pulse threshold fluence and incubation coefficients for all materials through line ablation experiments. As observed for materials with low incubation coefficient such as PLA and PET, a low threshold fluence can lead to increased material removal which can inhibit microstructure formation.

This study confirmed the possibility of porous network formation on several polymers at different machining conditions. Furthermore, several guidelines with regards to their design were given which significantly contributes to future optimization studies.

\section{ACKNOWLEDGEMENTS}

This research has been funded by the National Sciences and Engineering Research Council of Canada (NSERC) and the McGill Engineering Doctoral Award (MEDA).

\section{REFERENCES}

[1] Goddard, J. M. and Hotchkiss, J. H., "Polymer surface modification for the attachment of bioactive compounds," Prog. Polym. Sci., 32(7), 698-725, (2007).

[2] Martinez, A. W. and Chaikof, E. L., "Microfabrication and nanotechnology in stent design," Wiley Interdiscip. Rev. Nanomed. Nanobiotechnol., 3(3), 256-268, (2011).

[3] Rashid, S. T., et al., "Engineering of bypass conduits to improve patency," Cell Prolif., 37(5), 351-366, (2004).

[4] Govindarajan, T. and Shandas, R., "A Survey of Surface Modification Techniques for Next-Generation Shape Memory Polymer Stent Devices," Polym., 6(9), 2309, (2014).

[5] del Campo, A. and Arzt, E., "Fabrication Approaches for Generating Complex Micro- and Nanopatterns on Polymeric Surfaces," Chem. Rev., 108(3), 911-945, (2008).

[6] Shirk, M. D. and Molian, P. A., "A review of ultrashort pulsed laser ablation of materials," J. Laser Appl., 10(1), 1828, (1998).

[7] Bäuerle, D., [Laser processing and chemistry], Springer, Berlin \& New York, (2000).

[8] Lippert, T., et al., "Laser induced chemical and physical modifications of polymer films: dependence on the irradiation wavelength," Appl. Surf. Sci., 109-110227-231, (1997).

[9] Küper, S. and Stuke, M., "UV-excimer-laser ablation of polymethylmethacrylate at $248 \mathrm{~nm}$ : Characterization of incubation sites with Fourier transform IR- and UV-Spectroscopy," Appl. Phys. A, 49(2), 211-215, (1989).

[10] Adhi, K. P., et al., "Femtosecond ultraviolet (248 nm) excimer laser processing of Teflon (PTFE)," Appl. Surf. Sci., 218(1-4), 17-23, (2003).

[11] Hashida, M., et al., "Non-thermal ablation of expanded polytetrafluoroethylene with an intense femtosecond-pulse laser," Opt. Express, 17(15), 13116-13121, (2009).

[12] Huang, M. Z. and Ming, Z., "Femtosecond laser on the surface of PTFE," J. Func. Mater., (2010).

[13] Baset, F., et al., "Femtosecond laser induced porosity in poly-methyl methacrylate," Appl. Surf. Sci., 282729-34, (2013).

[14] Guay, J. M., et al., "Polarization-dependent Femtosecond Laser Ablation of Poly-methyl Methacrylate," N. J. Ph., 14(8), 085010 (17 pp.), (2012).

[15] Kruger, J., et al., "Femto- and nanosecond laser treatment of doped polymethylmethacrylate," Appl. Surf. Sci., 247(14), 406-11, (2005).

[16] Baudach, S., et al., "Ultrashort pulse laser ablation of polycarbonate and polymethylmethacrylate," Appl. Surf. Sci., 154-155555-560, (2000).

[17] Liang, F., et al., "Robust Non-Wetting PTFE Surfaces by Femtosecond Laser Machining," Int. J. Mol. Sci., 15(8), 13681-13696, (2014).

[18] Ahmmed, K. M. T., et al., "Introducing a new optimization tool for femtosecond laser-induced surface texturing on titanium, stainless steel, aluminum and copper," Opt. L. E., 66(0), 258-268, (2015). 
[19] Schneider, C. A., Rasband, W. S., and Eliceiri, K. W., "NIH Image to ImageJ: 25 years of image analysis," Nat. Meth., 9(7), 671-675, (2012).

[20] Allain, C. and Cloitre, M., "Characterizing the lacunarity of random and deterministic fractal sets," Phys. Rev. A, 44(6), 3552-3558, (1991).

[21] Utrilla-Coello, R. G., et al., "Microstructure of retrograded starch: Quantification from lacunarity analysis of SEM micrographs," J. Food Eng., 116(4), 775-781, (2013).

[22] Ling, E. J. Y., Servio, P., and Kietzig, A.-M., "Fractal and Lacunarity Analyses: Quantitative Characterization of Hierarchical Surface Topographies," Microsc. Microanal., 22(01), 168-177, (2016).

[23] Biswas, S., Karthikeyan, A., and Kietzig, A.-M., "Effect of Repetition Rate on Femtosecond Laser-Induced Homogenous Microstructures," Mat., 9(12), 1023, (2016).

[24] Wright, D. C., [Failure of plastics and rubber products : causes, effects, and case studies involving degradation], Rapra Technology, Shawbury, (2001).

[25] Liu, J. M., "Simple technique for measurements of pulsed Gaussian-beam spot sizes," Opt. Lett., 7(5), 196-8, (1982).

[26] Jee, Y., Becker, M. F., and Walser, R. M., "Laser-induced damage on single-crystal metal surfaces," J. Opt. Soc. Am. B, 5(3), 648-659, (1988). 